\title{
ACE2 overexpression inhibits acquired platinum resistance-induced tumor angiogenesis in NSCLC
}

\author{
QIJIAN CHENG*, LING ZHOU*, JIANPING ZHOU, HUANYING WAN, QINGYUN LI and YUN FENG \\ Department of Respiration, Ruijin Hospital, School of Medicine, Shanghai Jiao Tong University, Shanghai 200025, P.R. China
}

Received February 27, 2016; Accepted April 9, 2016

DOI: $10.3892 /$ or.2016.4967

\begin{abstract}
Angiotensin II (AngII) is a multifunctional bioactive peptide in the renin-angiotensin system (RAS). Angiotensinconverting enzyme 2 (ACE2) is a newly identified component of RAS. We previously reported that ACE2 overexpression may inhibit cell growth and vascular endothelial growth factor (VEGF) production in vitro and in vivo. In the present study, we investigated the effect of ACE2 on tumor-associated angiogenesis after the development of acquired platinum resistance in non-small cell lung cancer (NSCLC). Four NSCLC cell lines, A549, LLC, A549-DDP and LLC-DDP, were used in vitro, while A549 and A549-DDP cells were used in vivo. A549-DDP and LLC-DDP cells were newly established at our institution as acquired platinum-resistant sublines by culturing the former parent cells in cisplatin (CDDP)-containing conditioned medium for 6 months. These platinum-resistant cells showed significantly higher angiotensin II type 1 receptor (AT1R), ACE and VEGF production and lower ACE2 expression than their corresponding parent cells. We showed that ACE2 overexpression inhibited the production of VEGF in vitro and in vivo compared to their corresponding parent cells. We also found that ACE2 overexpression reduced the expression of AT1R and ACE. Additionally, we confirmed that ACE2 overexpression inhibited cell growth and VEGF production while simultaneously suppressing ACE and AT1R expression in human lung cancer xenografts. Our findings indicate that ACE2 overexpression may potentially suppress angiogenesis in NSCLC after the development of acquired platinum resistance.
\end{abstract}

Correspondence to: Dr Yun Feng or Professor Qingyun Li, Ruijin Hospital, Shanghai Jiaotong University School of Medicine, 197 Ruijin Road II, Shanghai 200025, P.R. China

E-mail: fy01057@163.com

E-mail: liqingyun68@hotmail.com

*Contributed equally

Key words: NSCLC, ACE2, acquired platinum resistance, VEGF, AngII

\section{Introduction}

Lung cancer is the leading cause of cancer-related deaths worldwide, and non-small cell lung cancer (NSCLC) accounts for almost $80 \%$ of all lung cancers. Cisplatin (CDDP) is one of the most important cytotoxic agents for the treatment of advanced NSCLC. However, cancer cells often develop multiple mechanisms to overcome CDDP-induced DNA damage and apoptosis, leading to CDDP resistance (1), which is a major limitation to its clinical effectiveness. Tumorassociated angiogenesis after the development of acquired platinum resistance is important for tumor growth and metastasis $(2,3)$.

Angiotensin II (AngII), a biologically active octapeptide in the renin-angiotensin system (RAS), mediates its biological effect by binding to two subtypes of receptors, type 1 (AT1R) and type 2 (AT2R), that belong to the G-protein-coupled receptor superfamily (4). Angiotensin-converting enzyme 2 (ACE2) is a newly identified component of RAS, which converts AngII to angiotensin-(1-7) [Ang-(1-7)] (5), a peptide with vasodilator and antiproliferative properties. The tumor environment contains all RAS components necessary to produce angiotensin locally, in addition to systemically produced angiotensin. Previously, numerous prospective and retrospective studies have reported that cancer patients who received angiotensin-converting enzyme inhibitors (ACEIs) or angiotensin II type 1 receptor blockers (ARBs) had a longer survival than non-recipients (6-9). Studies have also shown that ACEIs and ARBs reduced cancer growth and angiogenesis in vitro and in vivo (10). Emerging data suggest that platinum-resistant cancer cells show significantly higher vascular endothelial growth factor (VEGF) expression than their corresponding parental cells in vitro and in vivo (2) and that acquired platinum resistance enhanced tumor angiogenesis through AT1R in bladder cancer (3). Our previous studies demonstrated that ACE2 overexpression reduced tumor-associated angiogenesis and tumor cell invasion in vitro and in vivo $(11,12)$. However, the role of ACE2 in tumor associated angiogenesis induced by acquired platinum resistance is still unknown.

Based on previous studies, we hypothesized that ACE2 plays an important role in tumor-associated angiogenesis induced by acquired platinum resistance. In the present study, we investigated the changes in VEGF and RAS component expression between platinum-resistant NSCLC cells and their corresponding parent cells in vitro and in vivo. The present 
study also examined the effect and signaling roles of ACE2 overexpression in acquired platinum resistance-induced tumor angiogenesis in NSCLC.

\section{Materials and methods}

Cell lines and culture. A549 lung cancer cells (Shanghai Institute of Cells) were maintained in Ham's F12 medium with $10 \%$ fetal bovine serum (FBS), $100 \mathrm{mg} / \mathrm{ml}$ penicillin and $100 \mathrm{U} / \mathrm{ml}$ streptomycin using media and growth reagents from Gibco-BRL (Grand Island, NY). Lewis lung carcinoma cells (LLC) (Shanghai Institute of Cells) were maintained in RPMI-1640 medium. To develop platinum resistance, A549 and LLC cells were supplemented with $10 \%$ FBS containing $\mathrm{CDDP}$, at $37^{\circ} \mathrm{C}$ in a humidified $5 \% \mathrm{CO}_{2}$ atmosphere. Then, the concentration of CDDP was increased to $3 \mu \mathrm{M}$. These cells were passaged upon reaching confluency over a 6-month period. These new cell lines were named A549-DDP and LLC-DDP (acquired platinum resistance for 6 months).

Retroviral vector construct and transduction. The pcDNA3.1 vector containing human ACE2 cDNA was kindly provided by Dr Paul McCray (University of Iowa, Iowa City, IA, USA). The vectors were ligated into the $B g I I I / X h o I$ site in the pMSCV plasmid, which expresses human ACE2. 293T cells were transfected with DNA ( $4 \mu \mathrm{g}$ pMD-gag-pol, $4 \mu \mathrm{g}$ pMD-VSVG and $4 \mu \mathrm{g}$ retroviral vector pMSCV-ACE2 or pMSCV) using the Lipofectamine 2000 reagent (Invitrogen Corp., Carlsbad, CA, USA). The replication retrovirus was collected after $48 \mathrm{~h}$ and used to infect A549-DDP cells using Polybrene (Sigma Corp., Cream Ridge, NJ, USA) followed by centrifugation. Infected cells were selected with $4 \mu \mathrm{g} / \mathrm{ml}$ puromycin for 2 weeks. The resistant clones were isolated by limiting dilution and dispatched to new dishes. The obtained cells were subsequently named vector and A549-DDP-ACE2 cells.

Tumor growth assays. Six-to eight-week-old BALB/c athymic nude mice (Chinese Academy of Sciences, Shanghai, China) were used. Animal experiments were performed in accordance with the institutional guidelines of the University Committee on the Use and Care of Animals. To investigate CDDP sensitivity, the mice were administered CDDP $(10 \mathrm{mg} / \mathrm{kg})$ intraperitoneally on day 21 after cancer cell implantation. The mice were divided into the following 5 groups: i) A549, ii) A549-CDDP, iii) A549-DDP, iv) A549-DDP-CDDP and v) A549-DDP-ACE2. Each mouse was inoculated in the flank with $1 \times 10^{6}$ A549 cells ( $\left.\mathrm{n}=6\right)$, A549-DDP cells infected with MSCV $(n=6)$ or A549-DDP cells infected with ACE2 $(n=6)$. Tumor growth was monitored at 3-day intervals by measuring the tumor diameters using a vernier caliper. Tumor volume was determined based on the following formula: Tumor volume $=\left[(\right.$ major axis $\left.) \times(\text { minor axis })^{2}\right] \times 1 / 2$. Following a 30-day follow-up period, the mice were euthanized and the tumors were removed. Harvested tumors were cut into 2 pieces, one of which was placed in liquid nitrogen and frozen at $-80^{\circ} \mathrm{C}$ and the other was fixed using $4 \%$ paraformaldehyde and then embedded in paraffin.

Real-time quantitative reverse transcription analysis. ACE, ACE2, AT1R and VEGF mRNA expression in A549,
A549-DDP, LLC, LLC-DDP and A549-DDP-ACE2 cells were examined by real-time quantitative reverse transcription-based polymerase chain reaction (qRT-PCR) Total RNAs were extracted from the cells with TRIzol reagent. RNA was treated with DNase (Promega, Madison, WI, USA) and complementary DNA was synthesized using a cDNA synthesis kit (Applied Biosystems, Foster City, CA, USA) according to the manufacturer's instructions. Fluorescence qRT-PCR was performed using the double-stranded DNA dye SYBR-Green (PCR Core Reagents, PE Biosystems, Warrington, UK) using an ABI PRISM 7300 system (Applied Biosystems). The SYBR-Green assay contained $1 \mu \mathrm{l} 10 \mathrm{X}$ SYBR-Green PCR buffer, $0.8 \mu \mathrm{l}$ deoxynucleoside triphosphate (dNTP) mixture, $0.1 \mu \mathrm{l}$ AmpErase UNG (1 U/ $\mu \mathrm{l}), 0.05 \mu \mathrm{l}$ AmpliTaq Gold DNA Polymerase (5 U/ $\mu \mathrm{l}), 1.2 \mu \mathrm{l} \mathrm{MgCl}{ }_{2}(25 \mathrm{mM}), 0.1 \mu \mathrm{l}$ forward and reverse primer $(20 \mu \mathrm{M}), 1 \mu \mathrm{l} \mathrm{cDNA}$ and $5.65 \mu \mathrm{l}$ double distilled $\mathrm{H}_{2} \mathrm{O}$. The following PCR program was used to determine gene expression: i) $50^{\circ} \mathrm{C}$ for $2 \mathrm{~min}$, ii) $95^{\circ} \mathrm{C}$ for $10 \mathrm{~min}$ and iii) 45 cycles of $95^{\circ} \mathrm{C}$ for $30 \mathrm{sec}$ for denaturation, $60^{\circ} \mathrm{C}$ for $30 \mathrm{sec}$ for annealing and $72^{\circ} \mathrm{C}$ for $30 \mathrm{sec}$ for extension. All of the data were analyzed using the ABI PRISM SDS 2.0 software (Perkin-Elmer, Wellesley, MA, USA). GAPDH was co-amplified to normalize for the RNA amount added to the reactions and the data were subjected to cycling threshold analysis using the $\Delta \mathrm{Ct}$ method. PCR was repeated at least three times. The following primers were used in the present study: i) ACE forward, 5'-CCGATCTGGCAGAACTTC-3' and reverse, 5'-GTGTTCCAGATCGTCCTC-3' and ii) ACE2 forward, 5'-CCACTGCTCAACTACTTTGAGCC-3' and reverse, 5'-CTTATCCTCACTTTGATGCT.

Western blot analysis. ACE, ACE2, AT1R and VEGF expression levels were measured in the A549, A549-DDP, LLC, LLC-DDP and A549-DDP-ACE2 cells. After being treated with protein assay reagent (Bio-Rad Laboratories, Hercules, CA, USA), soluble protein was separated on $10 \%$ SDS-polyacrylamide gels and transferred to polyvinylidene fluoride (PVDF) membranes (Millipore, Billerica, MA, USA). ACE2 (AF933; R\&D Systems, Minneapolis, MN, USA), AT1R $(1: 1,000)$, VEGF $(1: 1,000)$ (both from Santa Cruz Biotechnology, Santa Cruz, CA, USA) and $\beta$-actin monoclonal antibodies (1:10,000, Sigma Corporation, Frederick, MD, USA) were used to detect ACE2, AT1R, VEGF and $\beta$-actin proteins, respectively. The immunoreactive bands were visualized by ECL Plus reagent (Amersham Biosciences, Piscataway, NJ, USA). VEGF expression protein in the A549 cells was analyzed by Scion Image (Scion Corp., Frederick, MD, USA).

In vitro tube formation assay. Cellular angiogenesis was measured in vitro based on tube formation on an extracellular Matrigel in a 24-well plate as previously described (13). Briefly, 24-well plates were coated with $40 \mathrm{ml}$ cold liquid Matrigel $(8 \mathrm{mg} / \mathrm{ml}) /$ well and incubated at $37^{\circ} \mathrm{C}$ for $30 \mathrm{~min}$ to promote solidification. A549 or A549-DDP or A549DDP-ACE2 cells were grown for $48 \mathrm{~h}$ in FCS-free media prior to incubation with different fatty acids in the presence of $5 \%$ fetal calf serum (FCS) media. Cells were seeded at a density of 70,000 cells/well. After a $16 \mathrm{~h}$ incubation, the wells were photographed with an inverted microscope at a magnification of x4 (Nikon TS100F; Nikon Corporation, Tokyo, Japan). 
A

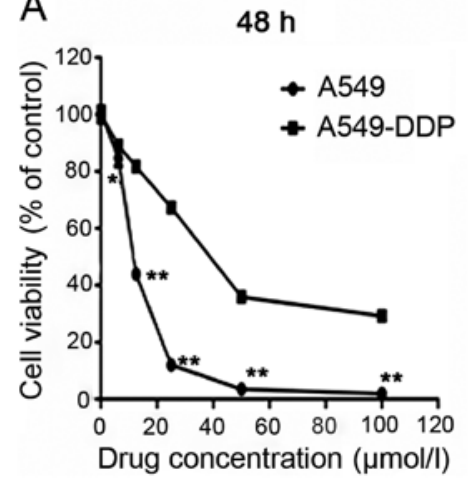

$\mathrm{B}$

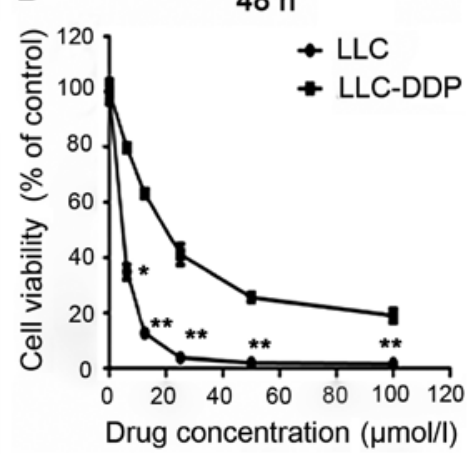

Figure 1. Comparison of drug resistance to CDDP in A549, A549-DDP, LLC and LLC-DDP cells. (A) A549 and A549-DDP cells were treated with different concentrations of CDDP for $48 \mathrm{~h}$. (B) LLC and LLC-DDP cells were treated with different concentrations of CDDP for $48 \mathrm{~h}$. The cell viability was determined by MTT assay ( $\mathrm{P}<0.05$ and $\left.{ }^{* *} \mathrm{P}<0.01\right)$.

Images were captured from the central view of at least five different fields/well and the extreme edges were excluded due to gel meniscus formation. Adobe Photoshop (version CS4) was used to quantify the tubule length from capillary network formation (14). The length of each tubule was determined by drawing a line over each tubule and the mean length of the lines (pixels) drawn in each image was calculated. Quantification of the tubular networks was measured by counting the total length of tubes in pixels. The results are expressed as a $\%$ over the control using the following formula: $\%$ over the control $=$ the mean length of total tubes $x$ 100/mean length of the tubes. The number of capillary connections or branch points between cells was counted manually. Independent experiments were performed in triplicate to reduce intra-assay variability. The average number and length of the connections were calculated for each fatty acid treatment.

\section{Results}

Establishment of the acquired platinum-resistant NSCLC sublines in vitro. Using A549 and LLC cells, we generated NSCLC sublines with an acquired resistance to platinum. These cells were successfully cultured for 6 months in CDDP-conditioned medium and were named A549-DDP and LLC-DDP. Following 3 months without CDDP-exposure, further examinations were performed. A549, LLC, A549-DDP and LLC-DDP cells were treated with various CDDP concentrations for $48 \mathrm{~h}$ (Fig. $1 \mathrm{~A}$ and $\mathrm{B}$ ). The $\mathrm{IC}_{50}$ values for A549 and A549-DDP cells were 10.8土0.5 and $39.6 \pm 1.8 \mu \mathrm{mol} / 1$, respectively. The resistance factor (RF) was 3.67. The $\mathrm{IC}_{50}$ values for LLC and LLC-DDP cells were $8.5 \pm 0.4$ and $23.3 \pm 2.1 \mu \mathrm{mol} / 1$, respectively. The RF for these cells was 2.74 .

Effect of acquired platinum resistance on VEGF production and RAS components in vitro. Analysis of the qRT-PCR data for ACE, ACE2, AT1R and VEGF mRNAs, which were corrected with GAPDH as an internal control, showed that both A549-DDP and LLC-DDP sublines showed significantly higher ACE, AT1R and VEGF mRNA expression (Fig. 2A, C and D) and lower ACE2 mRNA (Fig. 2B) expression than their corresponding parent cells. Using western blot analysis, both the A549-DDP and LLC-DDP sublines showed significantly higher ACE, AT1R and VEGF expression and lower ACE2 (Fig. 2E) expression than their corresponding parent cells.

Effect of ACE2 gene transfection on A549-DDP cells. Our objective was to determine the efficacy of the MSCV-ACE2 transfection. A549-DDP cells were infected with MSCV-ACE2 and selected in the presence of puromycin. Infection of A549-DDP cells with MSCV-ACE2 resulted in robust ACE2 expression by western blot analysis at $72 \mathrm{~h}$. Fig. 3A shows that ACE2 was overexpressed in the A549-DDP-ACE2 group in comparison with the A549-DDP group. As expected, the expression of ACE2 mRNA (Fig. 3B) was notably higher in the A549-DDP-ACE2 group when compared to the A549-DDP group.

Effect of ACE2 overexpression on VEGF production and $R A S$ components in vitro. We investigated whether the ACE2 overexpression inhibited VEGF production in acquired platinum-resistant NSCLC sublines. ACE, AT1R and VEGF mRNA expression was decreased in the A549-DDP-ACE2 group when compared to the A549-DDP group (Fig. 4A-C). Western blot analysis also demonstrated that the ACE, AT1R and VEGF productions was decreased in the A549-DDP-ACE2 group compared to the A549-DDP group (Fig. 4D).

Effect of ACE2 overexpression on tube formation in acquired platinum-resistant NSCLC sublines. A tube formation assay was performed by Matrigel to evaluate the angiogenic response of acquired platinum-resistant NSCLC sublines. A549-DDP cells significantly increased capillary network complexities and induced more tubes in the network compared with A549 cells. The A549-DDP-ACE2 cells showed a reduced ability to form capillary-like structures (i.e. decreased tube length and numbers of branches) compared to the A549-DDP cells (Fig. 5).

Effect of ACE2 overexpression on the PIBK/Akt and MAPK signaling pathways in the A549-DDP cells. The PI3K/Akt and MAPK signaling pathways are involved in the expression of VEGF and angiogenesis. To investigate the anti-angiogenic 

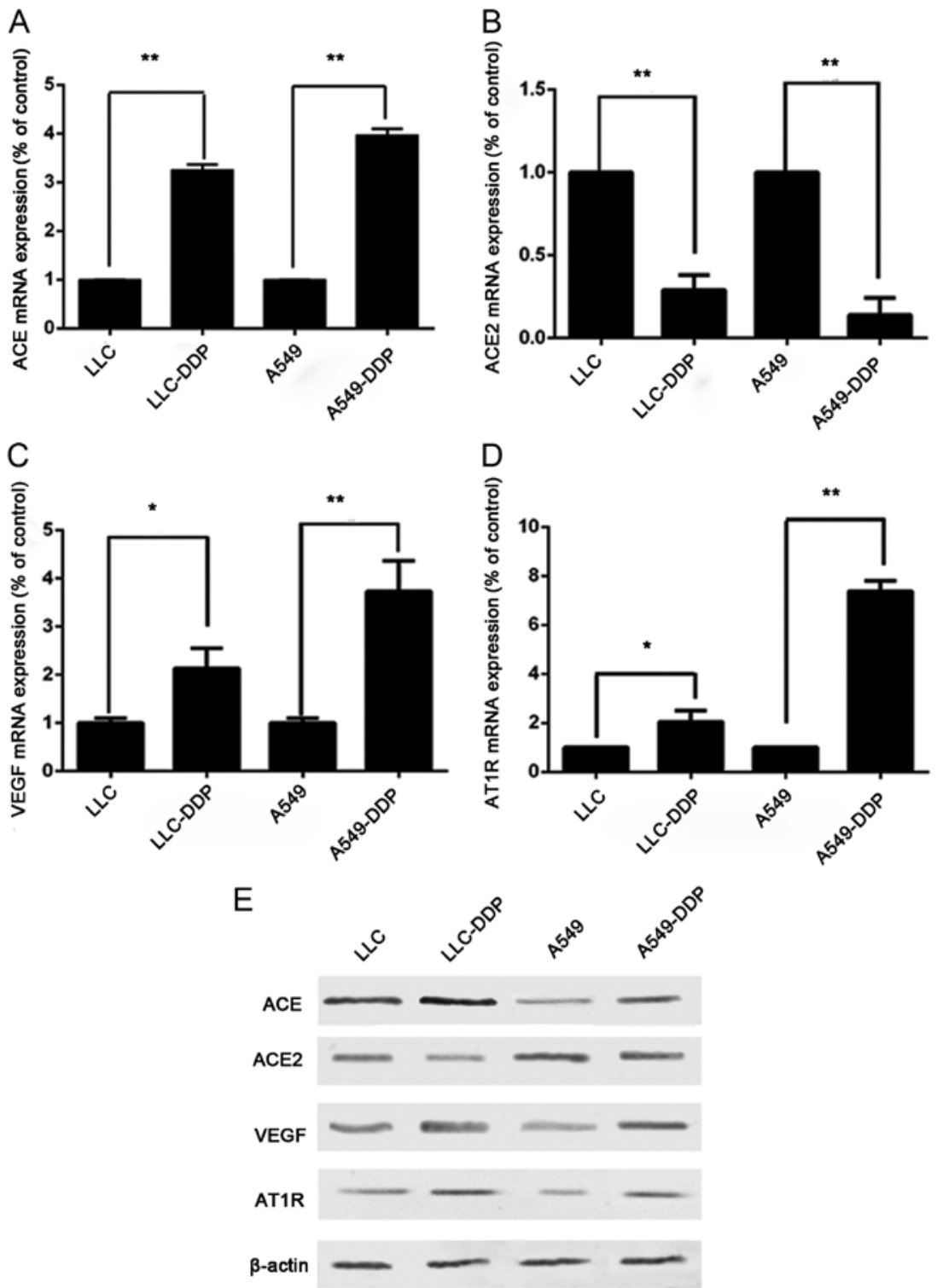

Figure 2. Effect of acquired platinum resistance on VEGF production and RAS components in vitro. (A-D) Effects of acquired platinum resistance on the production of (A) ACE, (B) ACE2, (C) VEGF and (D) AT1R mRNAs in the cultured A549-DDP and LLC-DDP cells when compared to the A549 and LLC cells (mean $\pm \mathrm{SD}, \mathrm{n}=3$; ${ }^{*} \mathrm{P}<0.05$ and ${ }^{* *} \mathrm{P}<0.01$ ). (E) Effects of acquired platinum resistance on the production of ACE, ACE2, VEGF and AT1R in the cultured A549-DDP and LLC-DDP cells when compared to the A549 and LLC cells.
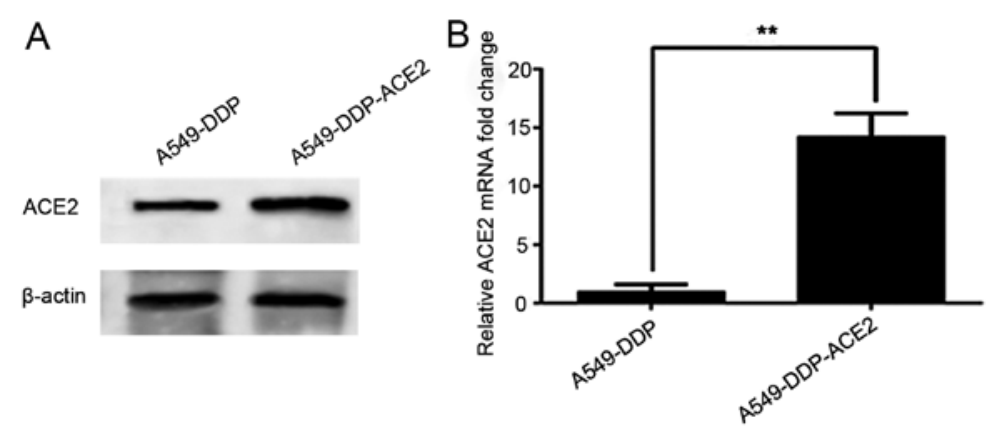

Figure 3. ACE2 expression was assessed in the A549-DDP and A549-DDP-ACE2 groups. (A and B) ACE2 expression was increased in the A549-DDP-ACE2 group when compared to the A549-DDP group by (A) western blot analysis and (B) real-time $\mathrm{PCR}$ (mean $\pm \mathrm{SD}, \mathrm{n}=3 ;{ }^{* *} \mathrm{P}<0.01$ ).

mechanism of ACE2 in the acquired platinum-resistant NSCLC sublines, the effects of ACE2 overexpression on the expression and phosphorylated status of PI3K/Akt and MAPK signaling proteins in A549-DDP cells were investigated by western 

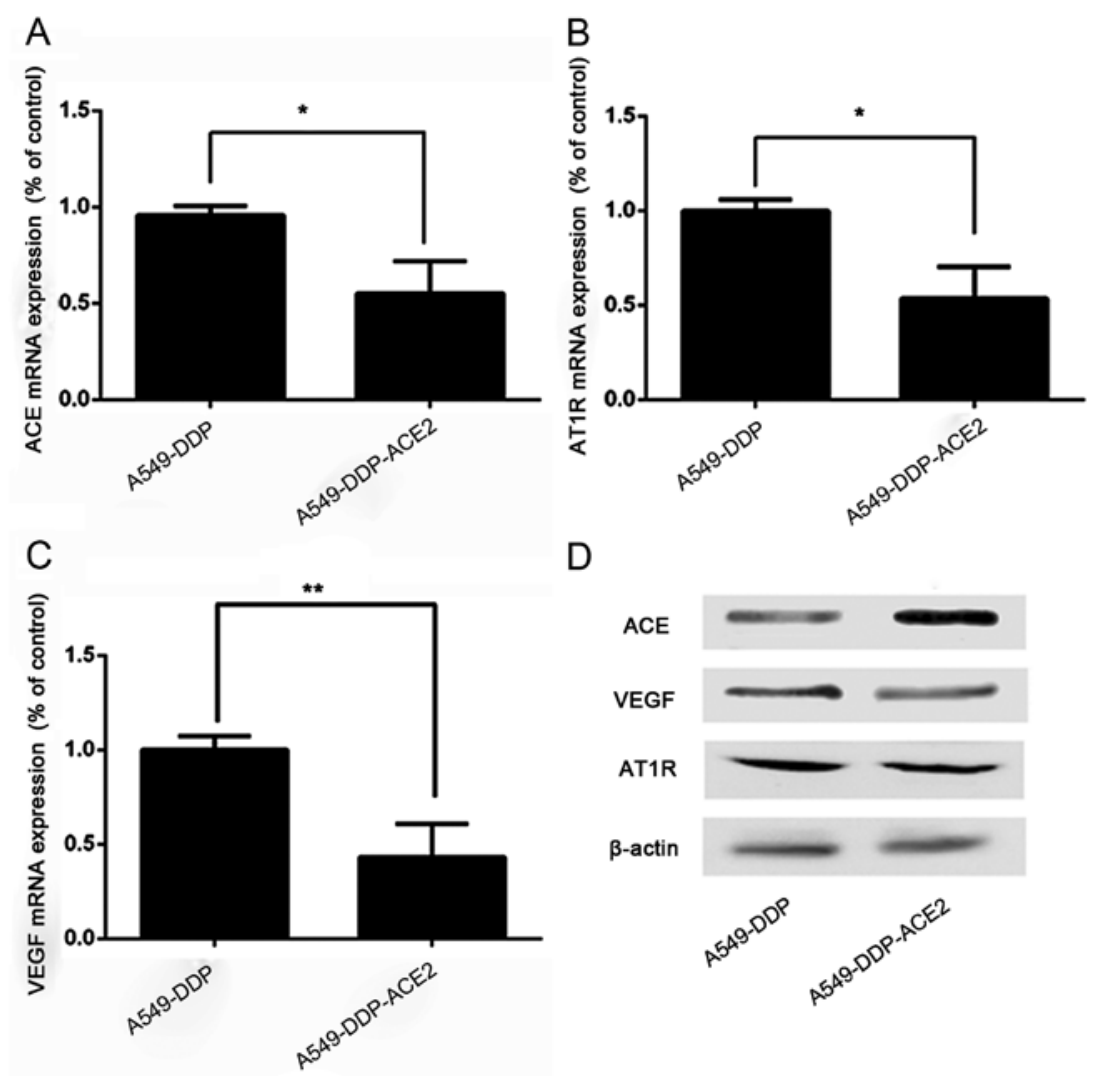

Figure 4. Effect of ACE2 overexpression on VEGF production and RAS components in vitro. (A-C) Effects of ACE2 overexpression on the expression of (A) ACE, (B) AT1R and (C) VEGF mRNAs in the A549-DDP-ACE2 cells when compared to the A549-DDP cells (mean \pm SD, $n=3$; ${ }^{*} \mathrm{P}<0.05$ and ${ }^{* *} \mathrm{P}<0.01$ ). (D) Effects of ACE2 overexpression on the production of ACE, VEGF and AT1R in the A549-DDP-ACE2 cells when compared to the A549-DDP cells.
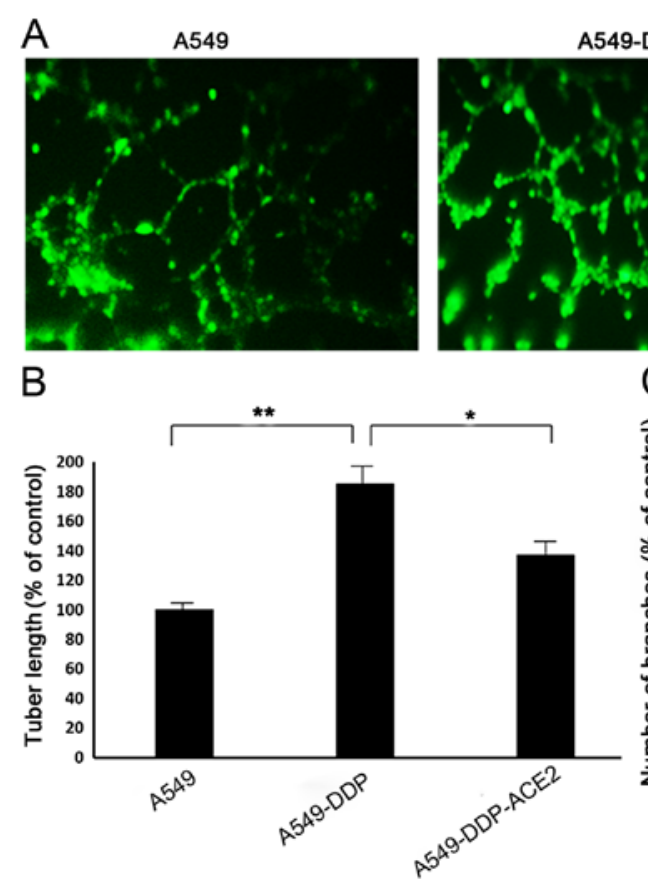
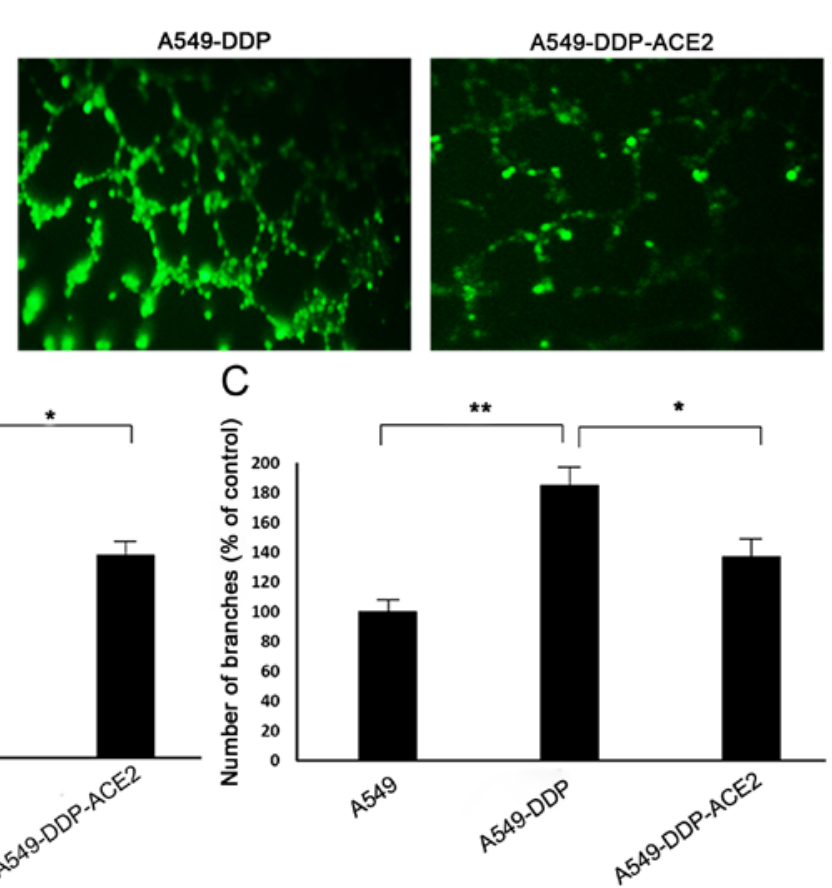

Figure 5. Effect of ACE2 overexpression on tube formation in the A549-DDP-ACE2 cells. (A) Representative images of tube formation in the A549, A549-DDP and A549-DDP-ACE2 cells cultured on Matrigel for $16 \mathrm{~h}$. (B) Tube length and (C) cell-branches were quantified. The results are expressed as the percentage of the tube length and branches in the A549 cells (mean $\pm \mathrm{SD}, \mathrm{n}=3$; ${ }^{*} \mathrm{P}<0.05$ and ${ }^{* *} \mathrm{P}<0.01$ ).

blot analysis (Fig. 6A). The levels of PI3K/Akt and MAPK signaling proteins were not altered in the A549-DDP cells, while the phosphorylation levels in PI3K/Akt and p38 MAPK signaling were significantly increased in A549-DDP cells 


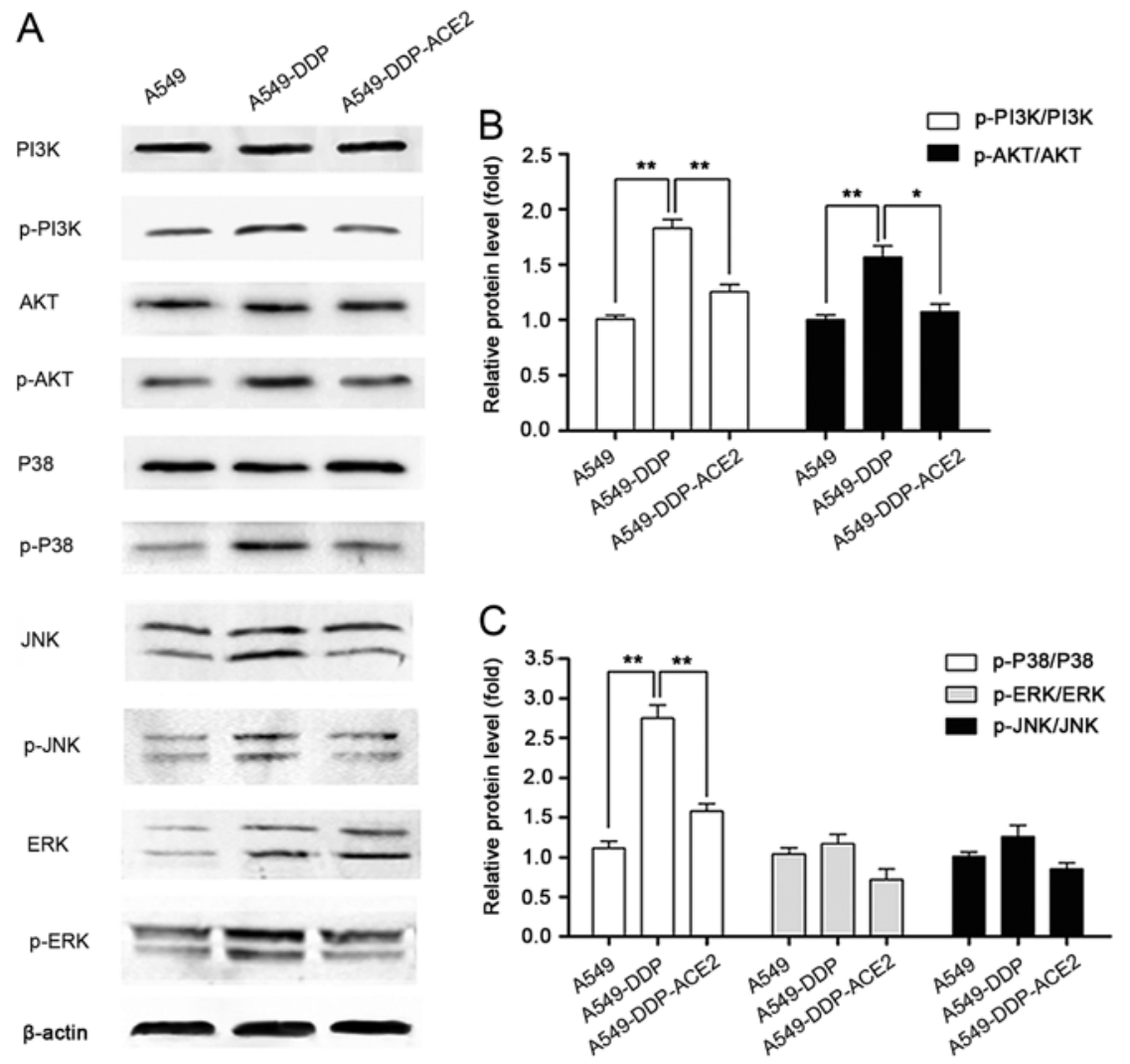

Figure 6. Effect of ACE2 overexpression on the PI3K/Akt and MAPK signaling pathways in A549-DDP cells. (A) The levels of proteins involved in PI3K/Akt (PI3K, phospho-PI3K, Akt and phospho-Akt) and MAPK signaling (p38, phospho-p38, JNK, phospho-JNK, ERK and phospho-ERK) were determined by western blot analysis. $\beta$-actin served as an internal control. (B) Densitometry showing p-PI3K and p-Akt levels in the A549, A549-DDP and A549-DDP-ACE2 cells. (C) Densitometry showing p-p38, p-ERK and p-JNK levels in the A549, A549-DDP and A549-DDP-ACE2 cells.
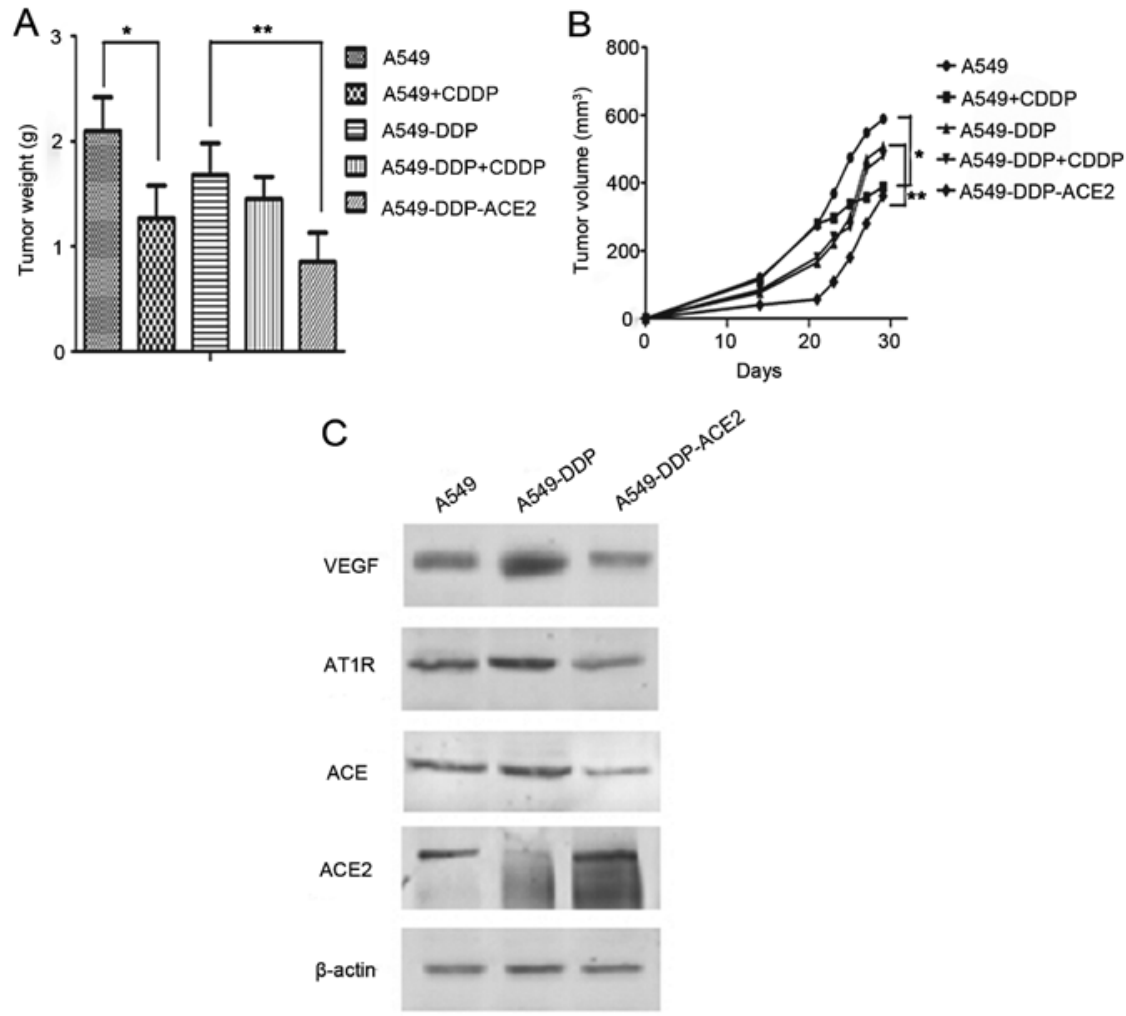

Figure 7. ACE2 overexpression inhibits the growth of A549-DDP tumor cells in vivo. The impact of ACE2 overexpression on (A) tumor weight and (B) volume is shown. The data points represent the mean \pm SD of 6 mice/group. Statistically significant differences are indicated in (A) for tumor weight and (B) for tumor volume ( $\mathrm{P}<0.05$ and $\left.{ }^{* *} \mathrm{P}<0.01\right)$. (C) Effects of ACE2 overexpression on the production of ACE, VEGF, ACE2 and AT1R in the A549-DDP-ACE2 cells when compared with the A549-DDP cells by western blot analysis in vivo. 
compared with A549 cells (Figs. 6B and C). In contrast, these active effects were blocked by the ACE2 overexpression in A549-DDP-ACE2 cells. The data revealed that inhibition of VEGF expression and angiogenesis in acquired platinumresistant NSCLC could partially occur through PI3K/Akt and p38 MAPK inactivation.

ACE2 overexpression inhibits the growth of A549-DDP tumor cells in vivo. To investigate whether the ACE2 overexpression inhibits pre-established tumor growth in nude mice, mice were divided into 5 groups (A549, A549-CDDP, A549-DDP, A549-DDP-CDDP and A549-DDP-ACE2). All of the animals were observed and tumors were measured during a 31-day follow-up period. Tumor weights in the A549-CDDP group were significantly reduced compared to the A549 group, while there were no significant changes in the A549-DDP group compared with the A549-DDP-CDDP group (Fig. 7A). The graph also showed that the tumor weight in the A549-DDP-ACE2 group was significantly reduced compared to the A549-DDP group. The data from the tumor growth curves are in agreement with the results for tumor weight and indicated that tumor growth in the A549-DDP-ACE2 group was significantly reduced when compared with the A549-DDP group (Fig. 7B). The results demonstrate that ACE2 overexpression significantly inhibited tumor growth in the acquired platinum-resistant A549-DDP nude mouse model. We next investigated whether ACE2 overexpression inhibits VEGF production in acquired platinum-resistant NSCLC sublines in vivo. By western blot analysis, A549-DDP cells showed significantly higher ACE, AT1R and VEGF expression and decreased ACE2 production than the A549 cells. The results also demonstrated that ACE, AT1R and VEGF production decreased in the A549-DDP-ACE2 group when compared to the A549-DDP group (Fig. 7C).

\section{Discussion}

In the present study, we analyzed RAS expression and VEGF production following the development of acquired platinum resistance in NSCLC. We also demonstrated the efficacy of ACE2 in acquired platinum resistance sublines in vitro and in vivo. To the best of our knowledge, this is the first study that shows the expression and effect of ACE2 on platinum-resistant cancer cells.

Current evidence supports the idea that both host and tumor RAS are important for tumor growth and angiogenesis in cancer (10,15). As a new part of RAS, the ACE2/Ang-(1-7)/Mas axis also plays an important role in cancer $(16,17)$. Recent studies have shown that low ACE2 expression may be a useful indicator of poor prognosis in HCC and gallbladder cancer $(18,19)$. Our previous study also demonstrated the same results (11).

In the present study, we established acquired platinum-resistant NSCLC sublines and found significantly higher ACE, AT1R and VEGF expression and lower ACE2 expression in both the A549-DDP and LLC-DDP sublines compared with their corresponding parent cells. A previous study also showed changes in AT1R and VEGF production after the development of acquired platinum resistance in bladder cancer (3). However, the present study focused more on the dysregulation of ACE/ACE2 in acquired platinumresistant NSCLC sublines. Notably, the ratio of ACE/ACE2 is important in RAS. Recent research has shown that an imbalance in this ratio leads to various diseases. Numerous studies have also shown that ACE2 appears to be a negative regulator of RAS and counterbalances the functions of ACE. ACE2 converts AngII to Ang-(1-7), which has the opposite function of AngII in cancer development. We previously found that Ang-(1-7) inhibits the migration and invasion of NSCLC cells (20).

We then overexpressed ACE2 in acquired platinum-resistant A549 cells and found that ACE2 overexpression altered the expression of other RAS components. We also found that ACE2 inhibited VEGF production and tumor-associated angiogenesis in NSCLC. To the best of our knowledge, this is the first study to show the effects of ACE2 and tumorassociated angiogenesis in cancer. Identifying the key proteins involved in these processes is vital for understanding carcinogenesis and for devising new therapies. The ACE2/Ang-(1-7)/ Mas axis, a new axis in RAS, has the opposite effect of the classical ACE/AngII/AT1R axis. ACE2, Ang-(1-7) and Mas have the same signaling pathways. A recent study showed that Ang-(1-7) suppressed hepatocellular carcinoma growth and angiogenesis through inactivation of the p38 MAPK phosphorylation signaling pathway (21). Our previous study also demonstrated that Ang- (1-7) inhibited migration and invasion through the PI3K/Akt and MAPK signaling pathways (20). In agreement with these studies, we observed that the effect of ACE2 on the inhibition of VEGF expression and angiogenesis in acquired platinum-resistant NSCLC partly occured through PI3K/Akt and p38 MAPK inactivation.

Our previous studies showed that the ACE2 overexpression inhibited cell growth and VEGF production in vivo $(11,12)$. A recent study also showed that ACE2 suppressed tumor growth in gallbladder cancer (22). In the present study, we found that ACE2 overexpression inhibited the growth of A549-DDP tumor cells in vivo. Consistent with our data, Tanaka et al found that ARB olmesartan suppressed tumor growth in acquired platinum-resistant tumors (3). Another previous study showed that Ang-(1-7) inhibited tumor angiogenesis in human lung cancer xenografts with a reduction in VEGF (23). We also demonstrated that ACE2 overexpression inhibited VEGF production in acquired platinum-resistant A549 cells. These data demonstrated that the effect of ACE2 on cell growth inhibition may be through the inhibition of VEGF production and tumor angiogenesis after the development of acquired platinum resistance.

In summary, acquired platinum resistance may induce tumor-associated angiogenesis and dysregulation of the local RAS system. Our studies showed that ACE2 overexpression could suppress VEGF expression in NSCLC and acquiredplatinum resistant NSCLC in vitro and in vivo. These results suggest the therapeutic potential of ACE2 for controlling tumor growth and tumor-associated angiogenesis after the development of acquired platinum resistance.

\section{Acknowledgements}

The present study was supported by the National Natural Science Foundation of China (nos. 81201837 and 81370130). 


\section{References}

1. Galluzzi L, Senovilla L, Vitale I, Michels J, Martins I, Kepp O, Castedo M and Kroemer G: Molecular mechanisms of cisplatin resistance. Oncogene 31: 1869-1883, 2012.

2. Pyaskovskaya ON, Dasyukevich OI, Kolesnik DL, Garmanchouk LV, Todor IN and Solyanik GI: Changes in VEGF level and tumor growth characteristics during Lewis lung carcinoma progression towards $c i s$-DDP resistance. Exp Oncol 29: 197-202, 2007.

3. Tanaka N, Miyajima A, Kosaka T, Miyazaki Y, Shirotake S, Shirakawa H, Kikuchi E and Oya M: Acquired platinum resistance enhances tumour angiogenesis through angiotensin II type 1 receptor in bladder cancer. Br J Cancer 105: 1331-1337, 2011.

4. Fleming I, Kohlstedt K and Busse R: The tissue renin-angiotensin system and intracellular signalling. Curr Opin Nephrol Hypertens 15: 8-13, 2006.

5. Arici M and Erdem Y: Dual blockade of the renin-angiotensin system for cardiorenal protection: An update. Am J Kidney Dis 53: 332-345, 2009.

6. Yoshiji H, Noguchi R, Toyohara M, Ikenaka Y, Kitade M, Kaji K, Yamazaki M, Yamao J, Mitoro A, Sawai M, et al: Combination of vitamin $\mathrm{K} 2$ and angiotensin-converting enzyme inhibitor ameliorates cumulative recurrence of hepatocellular carcinoma. J Hepatol 51: 315-321, 2009.

7. Nakai Y, Isayama H, Ijichi H, Sasaki T, Sasahira N, Hirano K, Kogure H, Kawakubo K, Yagioka H, Yashima Y, et al: Inhibition of renin-angiotensin system affects prognosis of advanced pancreatic cancer receiving gemcitabine. Br J Cancer 103: 1644-1648, 2010.

8. Wilop S, von Hobe S, Crysandt M, Esser A, Osieka R and Jost E: Impact of angiotensin I converting enzyme inhibitors and angiotensin II type 1 receptor blockers on survival in patients with advanced non-small-cell lung cancer undergoing first-line platinum-based chemotherapy. J Cancer Res Clin Oncol 135 $1429-1435,2009$

9. Tatokoro M, Fujii Y, Kawakami S, Saito K, Koga F, Matsuoka Y, Iimura Y, Masuda $\mathrm{H}$ and Kihara K: Phase-II trial of combination treatment of interferon- $\alpha$, cimetidine, cyclooxygenase- 2 inhibitor and renin-angiotensin-system inhibitor (I-CCA therapy) for advanced renal cell carcinoma. Cancer Sci 102: 137-143, 2011.

10. Imai N, Hashimoto T, Kihara M, Yoshida S, Kawana I, Yazawa T, Kitamura $\mathrm{H}$ and Umemura S: Roles for host and tumor angiotensin II type 1 receptor in tumor growth and tumor-associated angiogenesis. Lab Invest 87: 189-198, 2007.

11. Feng Y, Wan H, Liu J, Zhang R, Ma Q, Han B, Xiang Y, Che J, Cao $\mathrm{H}$, Fei $\mathrm{X}$, et al: The angiotensin-converting enzyme 2 in tumor growth and tumor-associated angiogenesis in non-small cell lung cancer. Oncol Rep 23: 941-948, 2010.
12. Feng Y, Ni L, Wan H, Fan L, Fei X, Ma Q, Gao B, Xiang Y, Che J and Li Q: Overexpression of ACE2 produces antitumor effects via inhibition of angiogenesis and tumor cell invasion in vivo and in vitro. Oncol Rep 26: 1157-1164, 2011.

13. Arnaoutova I, George J, Kleinman HK and Benton G: The endothelial cell tube formation assay on basement membrane turns 20: State of the science and the art. Angiogenesis 12: 267-274, 2009.

14. Moyes AJ, Maldonado-Pérez D, Gray GA and Denison FC: Enhanced angiogenic capacity of human umbilical vein endothelial cells from women with preeclampsia. Reprod Sci 18: 374-382, 2011.

15. Ino K, Shibata K, Kajiyama H, Nawa A, Nomura S and Kikkawa F: Manipulating the angiotensin system - new approaches to the treatment of solid tumours. Expert Opin Biol Ther 6: 243-255, 2006.

16. Ender SA, Dallmer A, Lässig F, Lendeckel U and Wolke C: Expression and function of the ACE2/angiotensin(1-7)/Mas axis in osteosarcoma cell lines U-2 OS and MNNG-HOS. Mol Med Rep 10: 804-810, 2014.

17. Fan L, Feng Y, Wan HY, Ni L, Qian YR, Guo Y, Xiang Y and Li QY: Hypoxia induces dysregulation of local renin-angiotensin system in mouse Lewis lung carcinoma cells. Genet Mol Res 13: 10562-10573, 2014.

18. Li J, Yang ZL, Ren X, Zou Q, Yuan Y, Liang L, Chen M and Chen S: ACE2 and FZD1 are prognosis markers in squamous cell/adenosquamous carcinoma and adenocarcinoma of gallbladder. J Mol Histol 45: 47-57, 2014.

19. Ye G, Qin Y, Lu X, Xu X, Xu S, Wu C, Wang X, Wang S and Pan D: The association of renin-angiotensin system genes with the progression of hepatocellular carcinoma. Biochem Biophys Res Commun 459: 18-23, 2015.

20. Ni L, Feng Y, Wan H, Ma Q, Fan L, Qian Y, Li Q, Xiang Y and Gao B: Angiotensin-(1-7) inhibits the migration and invasion of A549 human lung adenocarcinoma cells through inactivation of the PI3K/Akt and MAPK signaling pathways. Oncol Rep 27: 783-790, 2012.

21. Liu Y, Li B, Wang X, Li G, Shang R, Yang J, Wang J, Zhang M, Chen Y, Zhang Y, et al: Angiotensin-(1-7) suppresses hepatocellular carcinoma growth and angiogenesis via complex interactions of angiotensin II type 1 receptor, angiotensin II type 2 receptor and Mas receptor. Mol Med 21: 626-636, 2015.

22. Zong H, Yin B, Zhou H, Cai D, Ma B and Xiang Y: Loss of angiotensin-converting enzyme 2 promotes growth of gallbladder cancer. Tumour Biol 36: 5171-5177, 2015.

23. Soto-Pantoja DR, Menon J, Gallagher PE and Tallant EA: Angiotensin-(1-7) inhibits tumor angiogenesis in human lung cancer xenografts with a reduction in vascular endothelial growth factor. Mol Cancer Ther 8: 1676-1683, 2009. 